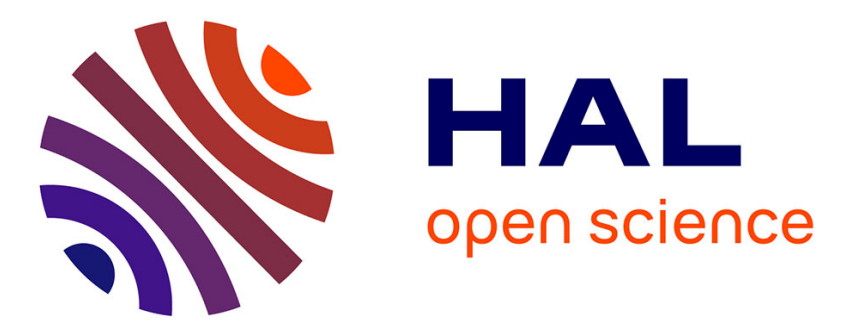

\title{
The spectrum of natural oscillations of an array of micro- or nanospheres on an elastic substrate
}

Victor A. Eremeyev, Elena A. Ivanova, Nikita F. Morozov, S. E. Strochkov

\section{To cite this version:}

Victor A. Eremeyev, Elena A. Ivanova, Nikita F. Morozov, S. E. Strochkov. The spectrum of natural oscillations of an array of micro- or nanospheres on an elastic substrate. Doklady Physics, 2007, 52 (12), pp.699-702. hal-00826529

\section{HAL Id: hal-00826529 https://hal.science/hal-00826529}

Submitted on 28 May 2013

HAL is a multi-disciplinary open access archive for the deposit and dissemination of scientific research documents, whether they are published or not. The documents may come from teaching and research institutions in France or abroad, or from public or private research centers.
L'archive ouverte pluridisciplinaire HAL, est destinée au dépôt et à la diffusion de documents scientifiques de niveau recherche, publiés ou non, émanant des établissements d'enseignement et de recherche français ou étrangers, des laboratoires publics ou privés. 


\title{
The Spectrum of Natural Oscillations of an Array of Micro- or Nanospheres on an Elastic Substrate
}

\author{
V. A. Eremeyev ${ }^{a, b, *}$ E. A. Ivanova ${ }^{c}$, Academician N. F. Morozov ${ }^{d}$, and S. E. Strochkov ${ }^{a}$
}

Investigation of the properties of nanodimensional biological objects and particles (nanoparticles) and their use in various fields, including medicine and pharmacology, are among the key directions in the development of nanotechnology [1, 2]. An important place among various nanoparticles belongs to those possessing spherical or nearly spherical shapes, such as fullerenes, dendrimers, micelles, vesicles, and liposomes [2-7]. Liposomes, micelles, and vesicles are widely used in modern medicine, for example, in order to deliver encapsulated drugs to target organs in the organism. Structurally, liposomes comprise spherical single- or multilayer lipid shells containing an internal liquid medium. Dendrimers, albeit possessing a more complicated structure, can also be considered as comprising a surface layer and a nucleus with different properties. Spherical nanoparticles are of interest not only in biology and medicine: numerous technological application examples are offered by fullerenes; dendrimers are promising materials for solar cells [5]; coating with micelles can be used for modification of the catalytic properties of surfaces [2]. The interior part of such nanoparticles can be used for the localization of substances that are difficult to retain under usual conditions $[2,3,5]$. Investigations into the mechanical and other physical properties of spherical nanoparticles encounter significant difficulties. Despite the long history of the investigation of cell membranes of micron dimensions from the standpoint of continuum mechanics and the theory of shells (see, e.g., [6, 8]), the properties of nanodimensional shells are still far from being completely clear.

\footnotetext{
a Southern Scientific Center, Russian Academy of Sciences, Rostov-on-Don, 344090 Russia

${ }^{b}$ Southern State University, Rostov-on-Don, 344090 Russia

${ }^{c}$ St. Petersburg State Polytechnical University,

St. Petersburg, 195251 Russia

${ }^{d}$ St. Petersburg State University, Petrodvorets,

St. Petersburg, 198204 Russia

*e-mail:eremeyev@math.rsu.ru; eremeyev_victor@mail.ru
}

The present study was devoted to the mechanical properties and structure of spherical nanoparticles in relationship to their natural oscillations. Several first eigenfrequencies of nanodimensional spherical particles can be determined from an analysis of the spectrum of natural oscillations of a substrate bearing an array of attached nanoparticles. Previously, we justified this approach [10-12], applied it to determining the properties of nanocrystals and nanotubes, and established the optimum experimental ratios of the geometric parameters of nanodimensional objects and substrates. In this study we have determined the spectrum of natural oscillations of an elastic plate (substrate) bearing an array of spherical shells. We propose a mechanical model that describes the oscillations of such a substrate with attached biological cells, organelles, or nanocapsules (micelles, liposomes) formed using artificial analogs of cell membranes. Note that the substrate can also represent a biological object such as a cell membrane. Questions pertaining to the adhesion of such nanodimensional objects were considered in $[2,5,9]$. A solution of the problem under consideration is of interest from the standpoint of ultrasonic-excitation-controlled behavior of nanospheres containing medicinal preparations - in particular, drug release at a resonance frequency - and modification of the properties of a surface covered with such micelles.

Cell membranes have rather complicated structures composed of lipids and proteins [6, 7]. From the standpoint of the present investigation, the mechanical properties of membranes can be described using the theory of shells [8]. In this study, the behavior of a cell (considered as a mechanical system) is treated within the framework of the theory of membrane shells, according to which the cell is modeled by a spherical shell that is loaded by an interior pressure. Since the intracell medium is liquid, it is assumed that the cell (capsule) can lose its shape as a result of the deformation, but the volume of liquid bounded by the shell surface remains constant. An analogous formulation of the problem is used in the theory of capillary phenomena [13].

1. Consider a rectangular elastic plate of length $a$, width $b$, and thickness $H$, to which $n$ identical spherical 


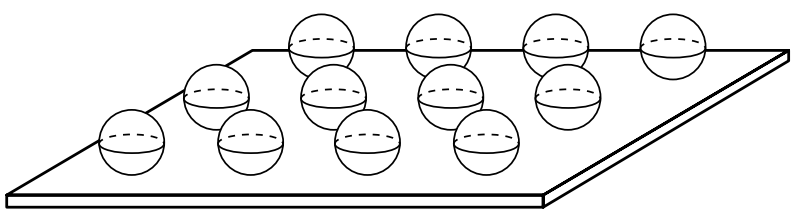

Fig. 1. Schematic diagram of an elastic plate with attached spherical shells.

elastic shells of thickness $h$ and radius $R$ are attached (Fig. 1). We assume that the shells are attached to the plate (substrate) at the points $x=x_{k}, y=y_{k}(k=1,2, \ldots$ $n)$. The spherical shells are considered as membranes loaded by a uniform internal hydrostatic pressure $p$. In the equilibrium state, the force acting on the shell surface is everywhere the same: $N_{1}=N_{2}=N=p R / 2$. This $N$ value is in fact the surface tension, and the whole membrane can be considered as a spherical shell of equal resistance [14].

The motion of a liquid inside the oscillating shell, as well as the wave propagating on the shell surface, will be ignored. In addition, lipid membranes are characterized by compressive-tensile deformations in the tangent plane, which are small compared to the bending deformations. The shell is described in a spherical coordinate system $(r, \varphi, \theta)$, where $\phi \in[0,2 \pi]$ and $\theta \in[0$, $\pi]$. The linearized oscillation equation for an expanded spherical membrane shell can be written as

$$
\begin{gathered}
\frac{N}{R^{2}}[\Delta u+2 u]+q+q_{k}=m \frac{\partial^{2} u}{\partial t^{2}}, \\
\Delta \equiv \frac{\partial^{2}}{\partial \theta^{2}}+\cot \theta \frac{\partial}{\partial \theta},
\end{gathered}
$$

where $m$ is the surface density of the shell, $u$ is the shell bending in the direction normal to the surface, $q$ is the additional pressure in the liquid interior, and $q_{k}$ is the force acting on the $k$ th shell from the substrate. Equation (1) is a partial case of the equation of motion used in the theory of prestrained shells with neglect of the bending and potential stiffness [15]. This equation describes small capillary oscillations of a spherical drop of a liquid with the surface tension $N$. Since all shells are assumed to be identical, the oscillations of each sphere are described by Eq. (1), differing only in the lumped load $q_{k}$ that depends on the point of attachment of the microsphere to the plate surface.

The isoperimetric condition of conservation of the liquid volume imposes the following limitation on the bending $u$ :

$$
\iint_{S} u d s=0
$$

where $S$ is the surface of the sphere. This integral relation is an additional equation that is necessary for deter- mining the pressure $q$ (considered as a response to the coupling (2)). As will be shown below, solutions to Eq. (1) can be expressed in terms of the Legendre polynomials, which automatically obey condition (2) owing to their mutual orthogonality. [15]

The equations of plate oscillations can be written as

$$
D \nabla^{4} w+\rho \frac{\partial^{2} w}{\partial t^{2}}=f, \quad \nabla^{2} \equiv \frac{\partial^{2}}{\partial x^{2}}+\frac{\partial^{2}}{\partial y^{2}},
$$

where $D$ is the bending stiffness, $\rho$ is the surface density, and $f$ is the transverse lumped load related to the presence of microspheres at the points $x=x_{k}, y=y_{k}(k=$ $1,2, \ldots, n)$. This load can be expressed as

$$
f=\sum_{k=1}^{n} q_{k} \delta\left(x-x_{k}, y-y_{k}\right),
$$

where $\delta(x, y)$ is the Dirac delta function of two variables. The condition of microsphere attachment to the plate surface leads to the kinematic relationships $\left.u_{k}\right|_{\theta=\pi}$ $=\left.w\right|_{x=x_{k}, y=y_{k}}$.

2. Let us consider the harmonic axisymmetric oscillations of a spherical shell described by Eqs. (1) and (2) under the action of a load lumped at the bottom point of the shell: $q_{k}=Q_{k} \delta(\theta-\pi) e^{i \omega t}$, where $\omega$ is the oscillation frequency. Nonaxisymmetric oscillation modes can be considered in an analogous manner.

Seeking for a solution to this problem in the form of $u=U(\theta) e^{i \omega t}$ and substituting $\cos \theta=\eta(\eta \in[-1,1])$, we can rewrite the equation of oscillations of the sphere as

$$
\frac{N}{R^{2}}\left[\frac{\partial}{\partial \eta}\left(1-\eta^{2}\right) \frac{\partial U}{\partial \eta}\right]+m \omega^{2} U+Q_{k} \delta(\eta+1)=0 .
$$

Expanding the solution to Eq. (4) formally into a series in orthogonal Legendre polynomials $P_{i}(\eta)$, we can express it as

$$
U(\eta)=\sum_{i=0}^{\infty} U_{i} P_{i}(\eta)
$$

where the coefficient $U_{0}$ must be zero in order to meet condition (2). The term $U_{1} P_{1}(\eta) \equiv U_{1} \cos \theta$ corresponds to the vertical motion of the sphere considered as an absolutely hard body.

Substituting expression (5) into Eq. (4) and using the well-known properties of the Legendre polynomials, we obtain the following expressions for the coefficients $U_{i}$ :

$$
U_{i}=\frac{(-1)^{n}}{4} \frac{2 i+1}{\frac{N[i(i+1)-2]}{R^{2}}-m \omega^{2}} Q_{k} \text {. }
$$




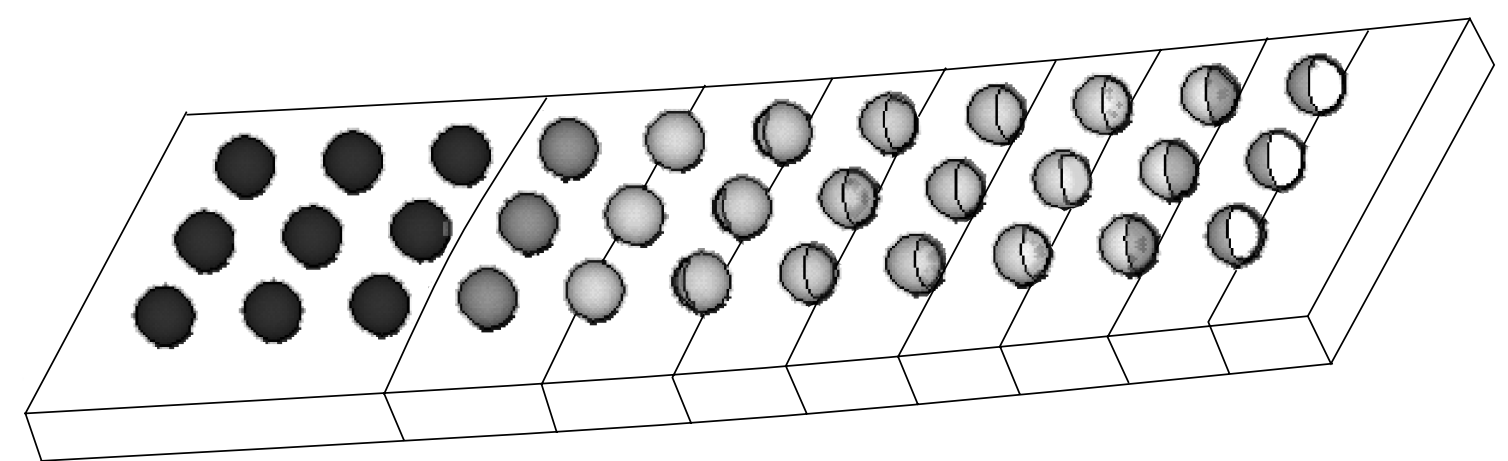

Fig. 2. The first bending mode of oscillations in the elastic plate.

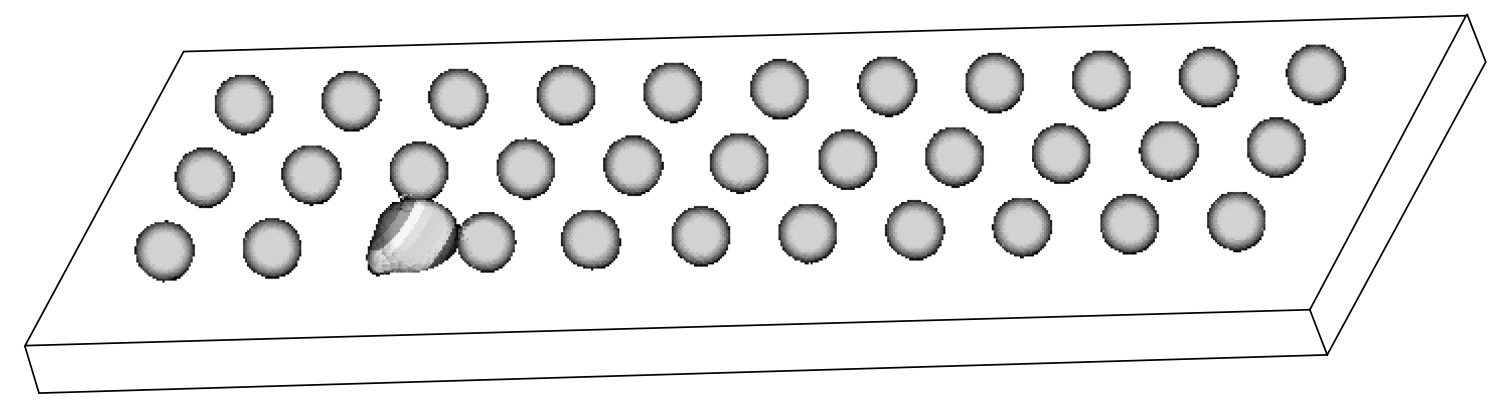

Fig. 3. The first (nonaxisymmetric) eigenmode localized in a nanosphere attached to the plate (depicted on an increased scale).

Note that series (5) with coefficients (6) is conditionally converging. Formula (6) implies the existence of the following set of eigenfrequencies:

$$
\omega_{i}^{*} \equiv \sqrt{[i(i+1)-2] \frac{N}{m R^{2}}},
$$

at which the sphere oscillates in the absence of an external load $\left(Q_{k}=0\right)$. The corresponding eigenmodes have the form of $U_{i} P_{i}(\eta)$.

3. Now let us consider oscillations of the plate, assuming that it has a pivoting support over the entire perimeter. For a large number of attached microspheres, we can assume that the transverse load is described by a continuous function of coordinates $(x, y)$. In other words, a microsphere is attached at every point of the plate surface, which produces a transverse load $f$ described by the equation

$$
-f=\frac{N}{R^{2}}[\Delta U+2 U]+\left.m \omega^{2} U\right|_{\eta=-1} .
$$

Seeking for a solution to Eq. (3) in the form of $w=$ $W(x, y) e^{i \omega t}$, using the representation $U=U_{l} P_{l}(\eta)$, and taking into account the kinematic relation $W(x, y)=U(-1) \equiv$ $(-1)^{l} U_{l}$, we can rewrite Eq. (3) as

$$
D \nabla^{4} W-\rho \omega^{2} W=\left[\frac{N}{R^{2}}[l(l+1)-2]-m \omega^{2}\right](-1)^{l} W .(7)
$$

This equation describes oscillations of a plate supported on an elastic inertial base of the Winkler type.

For a pivoting support, the function $W$ can be represented in the form of a Fourier series in sine functions as

$$
W=\sum_{i, j=1}^{\infty} W_{i j} \sin \frac{\pi i x}{a} \sin \frac{\pi j y}{b} .
$$

In these terms, Eq. (7) has nontrivial solutions under the following condition:

$$
\begin{aligned}
& D\left[\left(\frac{\pi i}{a}\right)^{2}+\left(\frac{\pi j}{b}\right)^{2}\right]^{2}-\rho \omega^{2} \\
= & (-1)^{n}\left[\frac{p R}{2} n(n+1)-m \omega^{2}\right] .
\end{aligned}
$$

Using Eq. (8), we can determine the set of eigenfrequencies for a plate covered with attached microspheres. Comparing the eigenfrequencies for the plates with and without attached particles, it is possible to determine some characteristics of microspheres, namely, their density and surface tension $N$ (or the internal pressure $p$ ).

4. A numerical analysis of the natural oscillations of a substrate covered with attached microspheres has been performed using the finite element method. All elements (substrate and microspheres) were considered 

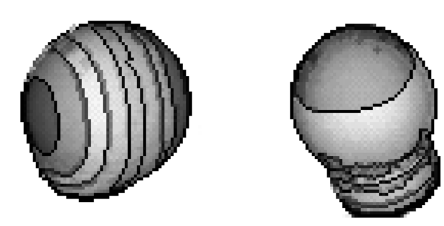

Fig. 4. The first and second eigenmodes localized in nanospheres.

as isotropic three-dimensional linear elastic bodies. The oscillation modes were analyzed for systems with various numbers of nanoparticles (ranging from one to 128), different geometries, and different types of substrate supporting. The nanoparticles were considered as hollow shells with a thickness two orders of magnitude smaller than the substrate thickness. For comparison, we have also considered the case of continuous nanoparticles (elastic balls).

In most cases, the results of calculations showed the localization of natural oscillations in the nanoparticles at frequencies corresponding to the first several eigenmodes of the attached shell. Figure 2 shows the first bending mode of a substrate with a bracket support, in which case the shells move without deformation. Figure 3 illustrates the case of nonaxisymmetric oscillations corresponding to the first eigenfrequency of the nanospheres, for which the plate remains virtually undeformed. Localized oscillation modes (analogous to that depicted in Fig. 2), in which the substrate is virtually immobile, have been also found for the second (axisymmetric) eigenfrequency. Figure 4 shows the first and second modes of the natural oscillations of nanospheres. More complicated modes of oscillations are observed at higher frequencies, for which both the substrate and nanospheres are deformed. Thus, the results of numerical calculations confirmed the validity of assumptions concerning the possibility of separating eigenfrequencies corresponding to a single nanodimensional object in the spectrum of a nanoparticle arraysubstrate system.

5. The theoretical and numerical analysis performed for nanospheres in this study, as well as that reported previously for nanocrystals and nanotubes [10-12], shows that it is possible to determine the eigenfrequencies of micro- and nanospheres in experiments using the measurement of eigenfrequencies of a substrate bearing an array of such nanodimensional objects attached to the surface. The eigenmodes of oscillations localized in the nanoparticles will be manifested by increased absorption of an acoustic wave with the corresponding frequency incident onto the substrate and by the detachment of nanoparticles (in the case of their weak adhesion) from an almost immobile substrate. These features in the behavior of a nanoparticle array- substrate system can also be used for the experimental determination of eigenfrequencies for continuous micro- and nanospheres.

\section{ACKNOWLEDGMENTS}

This study was supported in part by the Russian Foundation for Basic Research (project no. 05-0100o94), the Russian Science Support Foundation, and the Presidential Program for Support of Leading Scientific Schools in Russia (project no. NSh-4518-2006.1).

\section{REFERENCES}

1. Nanotechnology Research Directions: IWGN Workshop Report (Vision for Nanotechnology $R \& D$ in the Next Decade, Ed. by M. C. Roco, R. S. Williams and P. Alivisatos, (National Science and Technology Council-Kluwer Academic Publishers, New York 1999).

2. C. Poole, Jr. and F. Owens, Jr., Introduction. to Nanotechnology (John Wiley \& Sons, Hoboken (NJ, USA), 2003).

3. P. Harris, Carbon Nanotubes and Related Structures: New Materials for the Twenty-First Century (Cambridge University Press, Cambridge, 2001).

4. V. Balzani, A. Credi, and M. Venturi, Molecular Devices and Machines. A Journey into the Nano World, (WileyVCH, Weinheim, 2003).

5. Handbook of Nanoscience, Engineering, and Technology, Ed. by W. A. Goddard, D. W. Brenner, S. E. Lyshevski, and G. J. Iafrate (CRC Press, Boca Raton (USA), 2003).

6. R. B. Gennis, Biomembranes: Molecular Structure and Function (Springer-Verlag, New York, 1989).

7. R. Sato and Y. Kagawa, Transport and Bioenergetic in Biomembranes (New York, Plenum Press, 1982).

8. E. Evans and R. Skalak, Mechanics and Thermodynamics of Biomembranes (CRC Press, Boca Raton (USA), 1980).

9. J. Qian and H. Gao, Acta Biomater. 2, 51 (2006).

10. V. A. Eremeyev, E. A. Ivanova, N. F. Morozov, and A. N. Solov'ev, Dokl. Ross. Akad. Nauk 406, 756 (2006) [Dokl. Phys. 51, 93 (2006)].

11. V. A. Eremeyev, E. A. Ivanova, N. F. Morozov, and A. N. Solov'ev, Zh. Tekh. Fiz. 77, 3 (2007) [Tech. Phys. 52, 1 (2007)].

12. V. A. Eremeyev, E. A. Ivanova, N. F. Morozov, and S. E. Strochkov, Dokl. Akad. Nauk 415, 605 (2007) [Dokl. Phys. 52, 431 (2007)].

13. R. Finn, Equilibrium Capillary Surfaces (Springer-Verlag, New York, 1986).

14. S. P. Timoshenko, Plates and Shells (OGIZ GITTL, Moscow, 1948) [in Russian].

15. A. S. Vol'mir, Nonlinear Dynamics of Plates and Shells (Nauka, Moscow, 1972) [in Russian].

Translated by P. Pozdeev 